DOI: https://doi.org/10.11144/Javeriana.umed62-1.trau

\title{
Manejo no convencional del trauma penetrante cardiaco: una revisión integrativa de la literatura
}

\section{Non-Conventional Management of Penetrating Cardiac Trauma: An Integrative Literature Review}

Recibido: 25 Junio 2020 | Aceptado: 11 Diciembre 2020

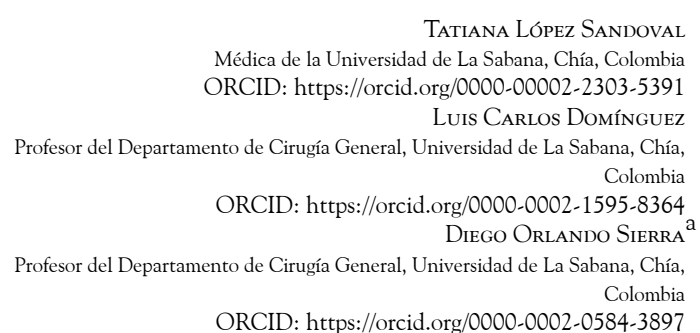

ORCID: https://orcid.org/0000-0002-0584-3897

a Autor de correspondencia: diego.sierra@unisabana.edu.co

Cómo citar: Lopéz Sandoval T, Domínguez LC, Sierra DO. Manejo no convencional del trauma penetrante cardiaco: una revisión integrativa de la literatura. Univ. Med. 2021;62(1). https://doi.org/10.11144/Jave riana.umed62-1.trau

\section{RESUMEN}

Introducción: En los últimos 30 años, la ventana pericárdica terapéutica se ha convertido en una alternativa para el abordaje de los pacientes con trauma penetrante cardiaco (TPC) sin afectación hemodinámica. Métodos: Revisión integrativa de la literatura, con búsqueda activa de artículos publicados a partir de 1980 hasta la actualidad, en inglés y español, en diferentes bases de datos que incluyeran en su descripción el manejo no convencional del TPC mediante ventana pericárdica y drenaje de hemopericardio, y que dentro de sus desenlaces evaluaran mortalidad, complicaciones postoperatorias o estancia hospitalaria. Resultados: Se identificó una mortalidad máxima del $63 \%$ en uno de los estudios incluidos en pacientes sometidos al quirúrgico abierto, por lesiones asociadas, así como una tasa hasta del $92 \%$ de esternotomías no terapéticas. Se resalta que las muestras de cada estudio fueron muy hterogéneas, así como las variables secundarias no incluidas en el análisis. Aunado a lo anterior, la tasa de complicaciones fue menor en el grupo de manejo no convencional comparado con los pacientes llevados a esternotomía/toracotomía, así como la estancia hospitalaria, respectivamente. Discusión: La evidencia demuestra que el manejo con ventana pericárdica y drenaje de hemopericardio en pacientes con TPC y estabilidad hemodinámica es seguro. La tasas de mortalidad complicaciones y estancia hospitalaria son inferiores con respecto al manejo convencional con esternotomía o toracotomía, aunque se deben tener en cuenta variables como el tiempo transcurrido entre el trauma y la atención, así como la persistente alta mortalidad de este tipo de lesiones, que las convierte en uno de los escenarios más desafiantes para el cirujano. Palabras clave

derrame pericárdico traumático; hemopericardio; trauma penetrante cardiaco; herida penetrante cardiaca; manejo conservador; manejo no operatorio; toracotomía emergente; esternotomía emergente. 


\begin{abstract}
Introduction: In the last 30 years, the therapeutic pericardial window has become an alternative for the approach of patients with Cardiac Penetrating Trauma (CPT) without hemodynamic compromise. Results: Seven studies were included: three experimental and four descriptive. Among their results, a maximum mortality of $63 \%$ was identified in one of the included studies, in patients submitted to open surgery, given mainly by associated lesions, as well as a rate of up to $92 \%$ of nontherapeutic sternotomies. It should be highlighted that the samples of each study were very heterogeneous, as well as secondary variables not included in the analysis of the same. In addition to the above, the rate of complications was lower in the non-conventional management group compared to patients who underwent sternotomy/ thoracotomy, as well as hospital stay, respectively. Discussion: Evidence shows that management with pericardial window and hemopericardial drainage in patients with SCT and hemodynamic stability is safe. Mortality rates of complications and hospital stay are lower than those of conventional management with sternotomy and/or thoracotomy, although variables such as time between trauma and care, as well as the persistently high mortality of this type of lesions should be taken into account, making it one of the most challenging scenarios for the surgeon.

Keywords

traumatic pericardial effusion; hemopericardium; penetrating cardiac trauma; penetrating cardiac injury; conservative management; non-operative management; emergent thoracotomy; emergent sternotomy.
\end{abstract}

\section{Introducción}

La herida abierta del corazón no es un tema nuevo. Fue descrita por primera vez en el año 3000 a. C. en el papiro de Edwin Smith y reforzadas con la muerte de Sarpedón en la renombrada Ilíada de Homero (1,2). Durante el siglo XVIII, Herman Boerhaave aseguró que todas las heridas cardiacas son fatales (3), lo cual fue cambiado en 1895, cuando Cappelen y Rhen repararon quirúrgicamente una herida de $1,5 \mathrm{~cm}$ de un ventrículo derecho humano (4). Fue hasta 1947 cuando Blalock reportó, posterior al conflicto de la Segunda Guerra Mundial, la primera pericardiocentesis diagnóstica y terapeútica para el manejo del taponamiento cardiaco (5).

Las heridas cardiacas se presentan con mayor frecuencia en hombres, hasta en el $91 \%$ de los casos (6). Como mecanismo de daño prevalente, se identifica la herida por arma cortopunzante hasta un $88 \%$ (6), seguido por las heridas por arma de fuego. Lo anterior ha venido cambiando en las últimas dos décadas, con el devenir de la venta de armas, lo que ha hecho que la prevalencia de ambas sea equiparable actualmente (7). En Colombia, para el 2019, la tasa de homicidios según las estadísticas del Instituto Nacional de Medicina Legal fue de 25,4 casos por cada 100.000 habitantes. La mayoría de los casos se presentó en Cali, Bogotá y Medellín (8). La amplia experiencia en en el manejo del trauma penetrante cardiaco (TPC) en el país ha permitido lograr tasas de supervivencia de hasta el $62 \%$, como la reportada por Duque et al. (9), muy similar a la reportada en Cali por Currea y Ferrada en 1995 (10). El área anatómica más afectada, en su mayoría por mecanismo cortopunzante, es el ventrículo derecho (39\%-43 $\%)$, seguido por el ventrículo izquierdo (33\%) y las cámaras atriales $(18 \%)(6,7,10)$.

Aun cuando Saadia et al., en 1994, clasificaron en cinco grupos a los pacientes del TPC según su presentación clínica (11), hoy en día su abordaje sistemático se hace según la presencia o no de signos de inestabilidad hemodinámica según el algoritmo de la Asociación Occidental de Trauma (12). Los pacientes con estabilidad hemodinámica, lesiones superficiales o trauma penetrante de bajo grado (I-III OIS AAST) pueden llegar a representar de un $20 \%$ a dos tercios de los pacientes que llegan con vida al hospital $(13,14)$. La clasificación de las heridas cardiacas, establecida por la Asociación Americana de Cirugía de Trauma (AAST), se expone en el anexo (10).

Clásicamente, los algoritmos terapéuticos para el manejo del TCP establecen que ante una ventana ecográfica positiva, el paciente debe ser conducido a esternotomía o toracotomía anterolateral izquierda, según la altura de la lesión, en los pacientes que se presentan con inestabilidad hemodinámica, y a ventana pericárdica en el paciente estable con subsecuente abordaje invasivo en el caso de encontrar hemopericardio (12). Así es como la ventana pericárdica ha tomado protagonismo, como un procedimiento menos invasivo en pacientes con ventana ecográfica positiva y estabilidad hemodinámica, 
en quienes usualmente se documentan heridas cardiacas de bajo grado, las cuales presentan sangrado limitado y ello disminuye las probabilidades de nuevo hemopericardio posdrenaje y taponamiento cardiaco subsecuente (15). Es posible, entonces, mediante la ventana pericárdica, la liberación del hemopericardio, la verificación del cese de sangrado activo y el corroborar la lesión miocárdica, de ser posible según la ubicación, o por medio del método recientemente publicado: el uso de fibras ópticas o asistencia toracoscópica (16). El advenimiento de este tratamiento no convencional ha permitido disminuir la tasa de esternotomía no terapéutica, descrita hasta en el $78 \%$ de los abordajes $(15,17)$, y sus complicaciones (infección de sitio operatorio, septicemia de origen esternal y lesiones iatrogénicas durante el acto quirúrgico) $(13,17)$. No obstante, la aceptación de este tipo de tratamiento entre los cirujanos ha sido escasa, y muchos de ellos siguen prefiriendo una esternotomía o toracotomía ante una herida cardiaca con ventana pericárdica o ecográfica positiva $(16,18)$.

En este estudio se realizó una revisión integral de la literatura acerca del manejo operatorio no convencional (ventana pericárdica) en los pacientes estables hemodinámicamente con herida precordial penetrante, con el objetivo de evaluar su seguridad clínica en términos de mortalidad, complicaciones y estancia hospitalaria.

\section{Metodología}

Las revisiones integrales de la literatura son un tipo de revisión descriptiva que permite la caracterización y el análisis de estudios tanto cualitativos como cuantitativos, de diferentes índoles teóricas y metodológicas; además de crear críticas de la evidencia, establecer síntesis de la información disponible y presentar teorías potenciales para el continuo desarrollo de la investigación clínica (19). Se diferencia de los metanálisis y de las revisiones sistemáticas, pues, en el primer caso, se analizan estadísticamente estudios primarios con el objetivo de validar la aplicabilidad de sus resultados, y en el caso de las revisiones sistemáticas, estas incluyen únicamente estudios experimentales de carácter cuantitativo, con un análisis multivariable con intención comparativa (19). En este estudio se escogió una revisión integral para abordar cronológicamente los avances y desenlaces del manejo no convencional del TPC en pacientes adultos mediante ventana pericárdica y drenaje de hemopericardio, a partir de estudios originales (por ejemplo, experimentos clínicos, estudios observacionales y estudios de cohorte). Se excluyeron reportes de caso, editoriales, revisiones de tema y cartas editoriales, así como estudios sobre trauma cardiaco cerrado y otros sobre el manejo avanzado del TCP mediante abordaje convencional exclusivamente (esternotomía o toracotomía). Se revisaron estudios publicados a partir de 1980, en inglés y español, de carácter descriptivo y analítico, que reporten alguno de los siguientes desenlaces: mortalidad, complicaciones postoperatorias y estancia hospitalaria.

Se realizó una búsqueda de artículos relevantes en bases de datos como PubMed, Science Direct y Clinical Key, Cochrane y Lilacs, utilizando las siguientes palabras clave. derrame pericárdico traumático (traumatic pericardial effusion), hemopericardio (haemopericardium), trauma penetrante cardiaco (penetrating cardiac trauma), herida cardiaca penetrante (penentrating cardiac wound), tratamiento conservador (conservative treatment), manejo no operatorio (non-operative management), toracotomía emergente (emergency thoracotomy), esternotomía emergente (emergency sternotomy) y ventana pericárdica (pericardial window).

\section{Resultados}

En la búsqueda inicial se identificaron 2400 referencias. La selección a través del título y el resumen permitió identificar 36 artículos, de los cuales se excluyeron referencias duplicadas y estudios sobre trauma cerrado, trauma torácico extracardiaco y politraumatismo. La búsqueda inicial resultó en 14 estudios. 
De estos, se excluyeron cuatro estudios que evaluaban el rendimiento clínico de distintas pruebas diagnósticas en pacientes estables con TPC, como la ecografía de saco pericárdico y la tomografía axial computarizada, además de un reporte de caso del manejo conservador en soldados con misiles retenidos intracardiacos, una revisión histórica de la literatura y, finalmente, un estudio de predictores clínicos positivos en ventana pericárdica tanto en pacientes inestables como estables hemodinámicamente.

En total se incluyeron siete estudios (en inglés y español), tres experimentales y cuatro descriptivos, desde 1984 hasta 2020, en los cuales se evaluó el manejo no convencional en pacientes estables con TCP, en el que se analizará al menos uno de los desenlaces propuestos en la presente revisión (figura 1).

\section{Figura 1.}

Diagrama de flujo selección de estudios. En total 7 estudios fueron incluidos en la revisión

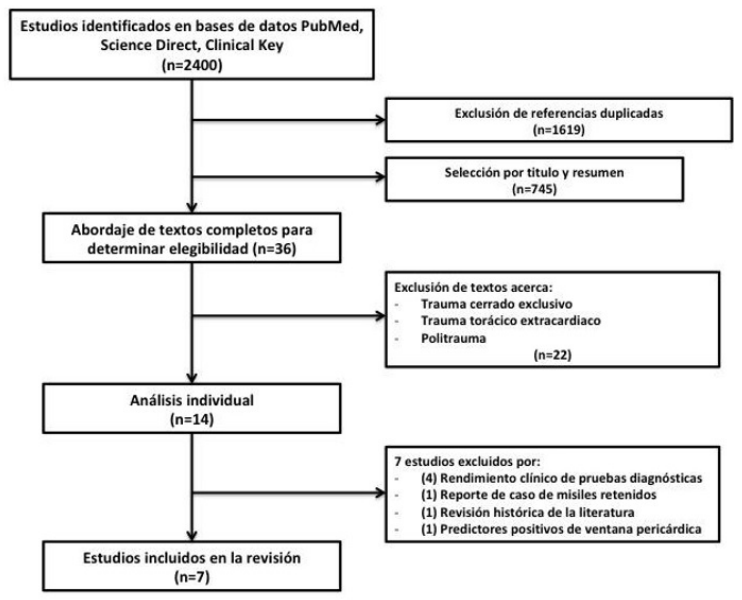

Evolución del manejo no convencional del TCP mediante ventana pericárdica

El manejo no convencional de los pacientes con TCP y estabilidad hemodinámica se originó en estudios desarrollados hace cuarenta años, los cuales buscaban evaluar el rendimiento diagnóstico de la ventana pericárdica. Esta última fue, por primera vez, descrita por Arom et al. (20), en 1977, quienes documentaron
50 pacientes con sospecha de taponamiento cardiaco, los cuales fueron operados a través de este nuevo abordaje. Los autores reportaron el hallazgo de hemopericardio en 46 pacientes en quienes se les realizó únicamente drenaje del hemopericardio, y en los cuatro pacientes restantes identificaron lesiones extracardiacas. No tuvieron mortalidad secundaria a la intervención; además, demostraron una disminución de la mortalidad global por el TCP, al tener cifras de un $8 \%$ en comparación con series anteriores, del $12 \%$, entre 1973 y 1976.

Posteriormente, Garrison et al. (21) describieron la ventana pericárdica transdiafragmática ante la sospecha de TPC en pacientes con trauma penetrante toracoabdominal que fueron llevados a laparotomía. En 1984, publicaron un estudio observacional retrospectivo con recolección de datos durante 7 años. Describieron la realización de ventana pericárdica transdiafragmática en 60 pacientes, 44 de ellos debido a proximidad anatómica de la lesión y 13 por inestabilidad hemodinámica no explicada por los hallazgos de la laparotomía. Identificaron 19 ventanas positivas (31\%), de las cuales $14 \quad$ (73 $\%)$ fueron llevadas a esternotomía; 3 (15 $\%)$, a toracotomía anterolateral izquierda, y 2 (10\%), a una extensión anterior de la incisión de la pericardiotomía. Como hallazgos intraoperatorios, 17 (89\%) pacientes presentaron heridas miocárdicas (especialmente en el ventrículo izquierdo), y otros 2 , heridas en el pericardio en quienes la cirugía no fue terapéutica. Reportaron una mortalidad global del $20 \%$ (21).

El concepto ventana pericárdica diagnóstica fue reforzado por Andrade et al. (14), quienes describieron retrospectivamente el manejo de 76 pacientes (1991-1992) con lesión penetrante en el área precordial y estabilidad hemodinámica, sometidos a una ventana pericárdica subxifoidea. El $83 \%$ de las lesiones fueron por arma cortopunzante. De los 76 pacientes, 16 (21 $\%)$ presentaron ventana pericárdica positiva, por lo que fueron sometidos a esternotomía, con una tasa de morbilidad reportada del $12,5 \%$ 
derivada de complicaciones postoperatorias, principalmente pulmonares (14). Los autores no informaron sobre tasas de esternotomías no terapéuticas entre sus resultados, pero sí mencionaron una tasa del $0 \%$ de lecturas faslso negativas o falso positivas.

Tales estudios descriptivos sobre la ventana pericárdica diagnóstica contribuyeron a desarrollar un abordaje menos invasivo en pacientes con TPC para la determinación de hemopericardio activo, en el cual se establece si la lesión cardiaca presenta sangrado activo o no posterior al drenaje del hemopericardio y, por consiguiente, a la decisión de llevar al paciente a esternotomía o toracotomía. Por lo tanto, a partir de 1994, varios grupos de investigadores plantearon estudios dirigidos a comprobar la seguridad clínica del manejo no convencional en el TPC, comparado con la exploración abierta convencional. En especial, esos estudios se han realizado en pacientes estables hemodinámicamente, en quienes la presentación clínica, y ecográfica, sostienen la sospecha de lesión pericárdica exclusiva o de lesión miocárdica de bajo grado.

La ventana pericárdica terapéutica como manejo no convencional del TCP fue introducida por primera vez en un estudio tipo cohorte prospectivo presentado por Navsaria et al. (15), en 2001. Los autores revisaron los desenlaces clínicos de 21 pacientes con TPC y estabilidad hemodinámica. Siete pacientes fueron manejados satisfactoriamente con lavado y drenaje exclusivo de hemopericardio por vía subxifoidea y los 14 restantes fueron sometidos a ventana pericárdica con lavado y drenaje y posteriormente a esternotomía para la clasificación de la lesión cardiaca. De este último grupo, se evidenciaron 10 pacientes con lesión miocárdica grado I a III. Los autores consideraron que la esternotomía en estos pacientes fue no terapéutica.

\section{Mortalidad}

Al sumar los resultados de los estudios incluidos, se identificaron 18 muertes en total, que representan una tasa de mortalidad del 5,4\%, si se hace un análisis global. Entre los resultados se identificó que la mortalidad en pacientes sometidos al manejo convencional varió del 0 $\%$ al $63 \%$, si se toman los valores aislados analizados en la tabla 1, en comparación con los pacientes llevados a manejo no convencional con una tasa de mortalidad maxima del $33 \%$, aunque las muestras de cada uno fueron muy variables, con gran heterogeneidad en los subgrupos, así como variables secundarias no incluidas en su análisis. Es de resaltar que en la mayoría de casos de la mortalidad presentada el $94,5 \%$ estuvo en el grupo de pacientes llevados a esternotomía, y el 5,5\% en el grupo de manejo no operatorio. Ahora bien, es importante mencionar que la tasa de mortalidad más alta presentada en el estudio de Garrison et al. (21), en 1984 ( $\mathrm{n}=$ 12), solo una fue directamente relacionada con la herida cardiaca; mientras que las restantes estuvieron dadas por lesiones graves asociadas y no propiamente por el procedimiento quirúrgico.

En 2011, Chad et al. (17) desarrollaron un estudio descriptivo retrospectivo entre 1996 y 2011, en el cual revisaron 377 ventanas pericárdicas. Un total de 55 fueron positivas, por lo que los pacientes fueron sometidos esternotomía, $38 \%$ de la cuales fueron no terapéuticas. Cuatro de estos pacientes murieron (tres por lesiones neurológicas centrales y uno por lesiones intrabdominales concomitantes).

En 2014, Nicol et al. (13) publicaron el primer ensayo clínico aleatorizado en el Hospital Groote Shuur en Ciudad del Cabo, Sudáfrica. Incluyeron 111 pacientes con TPC y ventana pericárdica subxifoidea positiva (hemopericardio), quienes fueron divididos en dos brazos: 55 pacientes fueron sometidos a esternotomía, y 56, pacientes a lavado y drenaje pericárdico. Dentro del primer grupo se reportaron 51 esternotomías no terapéuticas, que representan el $92 \%$ de la muestra, y uno de los pacientes murió (1,8 \% en el subgrupo y $0,9 \%$ en el análisis global), debido directamente a una complicacion intraoperatoria del abordaje abierto. En el segundo grupo ninguno requirió reintervención quirúrgica y no se reportó mortalidad intrahospitalaria. 


\section{Complicaciones}

En total se presentaron 60 complicaciones en los estudios incluidos, de las cuales, el $56 \%(\mathrm{n}=34)$ se presentó en el grupo de pacientes sometidos a manejo convencional con esternotomía o toracotomía; la tasa de complicaciones en este grupo fue del 24,2\%. Andrade et al. (14) reportaron una tasa de morbilidad cercana al 12,5 $\%$, especialmente por atelectasias e infecciones pleurales. Navsaria et al. (15), por otra parte, reportaron una tasa de complicaciones del 35 $\%$, especialmente por neumonía y atelectasias. Igualmente, Chad et al. (17) reportaron una tasa de complicaciones del 3,6\%, debida a lesiones iatrogénicas, pericarditis infecciosa y empiema pleural. Finalmente, Nicol et al. (13) reportaron una tasa de complicaciones cercana al $46 \%$, secundaria a neumonía, atelectasias, edema pulmonar e infección.

En comparación, la tasa de complicaciones reportadas en el grupo de pacientes sometidos a manejo no convencional fue del 20,6\%. Andrade et al. (14) documentaron un único caso de infección del sitio operatorio entre los pacientes en quienes la ventana pericárdica fue negativa y, por tanto, no fueron conducidos a exploración abierta, lo que representa una tasa de morbilidad del 1,7\%. Navsaria et al. (15) informaron una tasa de complicaciones del $57 \%$, secundaria a neumonía y atelectasias, especialmente dado que su muestra era más pequeña. Chad et al. (17) reportaron una complicación postoperatoria entre los tres pacientes llevados a manejo no operatorio (33\%), quienes desarrollaron síndrome de dificultad respiratoria y septicemia, que los condujeron a la muerte al día 13 de hospitalización (17). Finalmente, Nicol et al. (13) informaron una tasa de complicaciones menor a su grupo control ya mencionado, con un total de 20 complicaciones (35\%), las cuales fueron principalmente de origen respiratorio, además de dos septicemias secundarias a infección del sitio operatorio y a hemotórax retenido.

\section{Estancia hospitalaria}

La estancia en unidad de cuidado intensivo (UCI) declarada en los estudios incluidos fue de 2,01 días (2-2,04 días) para el grupo llevado a manejo convencional y de 2,12 días (0,25-4 días) en el grupo de no convencional con ventana pericárdica. Nicol et al. (13) notificaron una estancia media de 2,04 días en el primer grupo de pacientes y de 0,25 días en el segundo $(p$ $<0,05)$; el $84 \%$ de los pacientes de manejo no convencional fueron egresados de UCI en el primer días postoperatorio, en comparación con el $20 \%$ de los pacientes sometidos a esternotomía o toracotomía. Así mismo, Chestovich et al. (22), en el 2018, reportaron una estancia en UCI no significativa entre los dos grupos (4 versus 2 días; $p=0,18$ ).

En cuanto a la estancia hospitalaria regular, en los estudios que incluyeron este desenlace en su análisis se encontró un promedio de 6,7 días (6,5-7 días) en el grupo de esternotomía, comparado con 3,7 días (4,5-5 días) de los pacientes que recibieron manejo no convencional. Andrade et al. (14) encontraron un promedio de días de hospitalización de 7,75 días en los pacientes llevados a esternotomía; mientras que los pacientes con ventana pericárdica negativa recibieron vigilancia intrahospitalaria durante 30 horas únicamente. Navsaria et al. (15) informaron una tasa promedio de 5,9 días (3-10 días) en el grupo de pacientes llevados a esternotomía, comparado con 4,7 días (3-8 días) en el grupo de lavado y drenaje. Adicionalmente, Nicol et al. (13) registraron una estancia hospitalaria significativamente más larga en el grupo de pacientes llevados a esternotomía, comparado con su grupo control de manejo no operatorio, con un promedio de 6,5 días vs. 4,1 días, respectivamente $(p<0,001)$. Por último, Chestovich et al. (22) informaron una estancia hospitalaria promedio de 7 días $(4-20$ días) para el grupo de pacientes llevados a esternotomía; mientras que el grupo de lavado y drenaje exclusivo permanecieron en el hospital en promedio durante 5 días (5-12 días). Los 
resultados de cada estudio según desenlace se expone de manera detallada en la tabla 1.

\section{Tabla 1}

Resultados estudios incluidos en la revisión

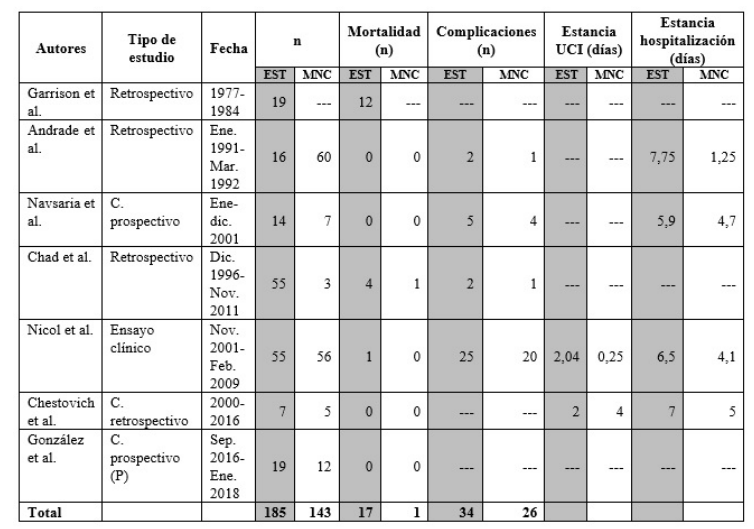

EST: esternotomía; MNC: manejo no convencional; UCI: unidad de cuidados intensivos

\section{Discusión}

En la presente revisión se identificó que el manejo no convencional con ventana pericardica y drenaje de hemopericardio en pacientes estables con TPC puede ser una opción terapéutica segura, pues ofrece una baja mortalidad baja, así como una incidencia de complicaciones posoperatorias y estancia hospitalaria global menor, en comparacion con el manejo convencional con esternotomía o toracotomía.

En la última década, varios estudios han respaldado la idea de incluir un nuevo enfoque para los pacientes con TPC, y no solamente determinar la conducta quirúrgica por la presencia/ausencia de hemopericardio. El ensayo clínico de Nicol et al. (13) demuestra resultados contundentes en cuanto a una tasa de mortalidad, morbilidad y estancia hospitalaria en los pacientes llevados a esternotomía. Así mismo, la cohorte retrospectiva de Chestovich et al. (22) aporta datos importantes, respecto a mortalidad, complicaciones y estancia hospitalaria; además, fue el primer estudio comparativo que demostró la incidencia de esternotomías no terapéuticas en pacientes estables hemodinámicamente, en quienes las lesiones encontradas intraoperatoriamente apoyaban al drenaje exclusivo del hemopericardio.

Los resultados expuestos en la presente revisión son similares a los presentados por grandes series mundiales del TPC con manejo quirúrgico convencional. Se debe tener en cuenta que todos los algoritmos avalados en trauma indican que todo paciente con ventana ecográfica positiva debe ser llevado a toracotomía o esternotomía con el propósito de evitar lesiones cardiacas inadvertidas $(12,23,24)$ en los casos más convencionales o, según la preferencia del cirujano, llevar al paciente a una ventana pericáridca, sobre todo si la ventana ecográfica no es concluyente, y de tener hallazgos intraoperatorios positivos para lesión miocárdica, conducir a un abordaje abierto. Una de las series más grandes de Estados Unidos en los últimos años, con 426 pacientes con TCP (2005-2014), demostró una mortalidad global del $51 \%$ en los pacientes llevados a esternotomía o toracotomía, y tasas de estancia hospitalaria similares a la encontrada en este estudio (25).

En Colombia, la tasa de mortalidad se encuentra entre el 2,5\% y el $13,5 \%$ de todos los pacientes sometidos a cirugía por TCP, con lesión cardiaca grado II y grado III, respectivamente (26), equiparable a lo presentado en el presente proyecto. En el país, el abordaje no convencional se utiliza con frecuencia en Cali. Los autores proponen, además, el uso de la ventana pericárdica toracoscópica (16) en pacientes con lesión pleural asociada, hemotórax o neumotórax que requiera toracostomía cerrada; así como ventanas pericárdicas por vía laparoscópica en pacientes con lesiones toracoabdominales concomitantes $(18,20)$. Lo anterior permite una mejor visualización de la superficie cardiaca, además de la posibilidad de sutura y reparación, en caso de ser necesaria, con resultados prometedores (18).

La presente revisión permite recopilar los datos disponibles, aunque limitados, en la literatura acerca del manejo no convencional del TCP, además de analizar globalmente diferentes desenlaces evaluados por los investigadores. En cuanto a límites de la presente revisión, se parte de que la mayoría de estudios 
incluidos son retrospectivos descriptivos, lo que limita sintetizar de manera concreta una posible recomendación basada en la evidencia, aunado a muestras pequeñas en los estudios experimentales disponibles y ausencia de información acerca de desenlaces a mediano y largo plazo. Se debe resaltar que los centros incluidos en los estudios ya mencionados son centros de referencia en trauma, por lo que variables como el tiempo transcurrido del trauma, así como la disponibilidad de infraestructura y tecnología para el monitoreo estricto de los pacientes llevados a manejo no convencional podrían tener descenlaces subrogados en poblaciones en las que no se logren garantizar.

Hacen falta mayores estudios clínicos aleatorizados para lograr establecer una conducta generalizable en la población. Sin embargo, los resultados de la presente revisión pueden ser considerados un punto de partida para la comunidad científica, en desarrollar nuevos modelos experimentales, y lograr incluir el manejo no convencional en los protocolos de trauma de diferentes instituciones.

Los algoritmos disponibles en trauma, hasta la fecha, indican la realización de esternotomía o toracotomía según la altura de la lesión, independiente del estado clínico del paciente, si se identifica derrame pericárdico en la ecografía de saco pericárdico o directamente en la ventana pericárdica. Sin embargo, la ventana pericárdica diagnóstica y terapéutica, junto con la ventana pericárdica por toracoscopia, se ha convertido en la alternativa mínimamente invasiva para el abordaje de los pacientes con trauma precordial penetrante con estabilidad hemodinámica. Nuestros resultados respaldan estas observaciones, tienen implicaciones para la práctica y ofrecen oportunidades de investigación.

\section{Referencias}

1. Gabrielli NM, Muñoz GS, Troncoso GP, Felmer EO, Kinast AC, Sánchez
HA, et al. Herida penetrante cardiaca. Cuad Cir. 2007;21:75-83.

2. Asensio JA, Soto SN, Forno W, Roldán G, Petrone P, et al. Penetrating cardiac injuries: a complex challenge. Surg Today. 2001;31:1041-53.

3. Lateef Wani $M$, Ahangar AG, Wani SN, Irshad I, Ul-Hassan N. Penetrating cardiac injury: a review. Trauma Monthly. 2012;17(1):230-2.

4. Crawford FA. Penetrating cardiac injuries. En: Sabiston DC, editor. Textbook of surgery: the biological basis of modern surgical practice. $14 . \cdot^{\text {a }}$ ed. Philadelphia: Saunders; 1991.

5. Ascensio JA, Ceballos JJ, Forno W, Gambaro E, Chahwan S, Marengo J, et al. Lesiones cardiacas penetrantes: una revisio\#n desde sus ori\#genes histo\# ricos hasta las u\#ltimas fronteras en el nuevo milenio. Parte 1. Cir Gen [internet]. 2000;22(1):81-90. Disponible en: https://www.medigraphi c.com/cgi-bin/new/resumen.cgi?IDAR TICULO $=2687$

6. Villegas LMI, Morales UCH, Rosero CE, Benítez EG, Cano RF, Fernández IM, et al. Trauma cardiaco penetrante: factores pronósticos. Rev Colomb Cir [internet]. 2007;22(3):148-56. Disponible en: https://www.redalyc.org /articulo.oa? $\mathrm{id}=35553447700815$

7. Bellister SA, Dennis BM, Guillamondegui OD. Blunt and penetrating cardiac trauma. Surg Clin North Am. 2017;97(5):1065-76.

8. Instituto Nacional de Medicina Legal y Ciencias Forenses. Boletín Estadístico Mensual. Forensis [internet]. 2019 ago;8. Disponible: https://www.medicinalegal .gov.co/documents/20143/349426/Bol etin + agosto $+2019 \cdot \mathrm{pdf} / 66 \mathrm{c} 6 \mathrm{f} 9 \mathrm{a} 0-8 \mathrm{e} 60$ -8a96-6d3d-b11a0999fcc7

9. Duque HA, Flórez LE, Moreno A, Jurado H, Jaramillo CJ, Restrepo MC. 
Penetrating cardiac trauma: followup study including electrocardiography echocardiography and functional test. World J Surg. 1999;23(12):1254-7.

10. Moore EE, Malangoni MA, Cogbill $\mathrm{TH}$, Shackford SR, Champion HR, Jurkovich GJ, et al. Organ injury scaling. IV: thoracic vascular lung cardiac and diaphragm. J Trauma. 1994;36:299-300.

11. Saadia R, Levy RD, Degiannis E, Velmahos GC. Penetrating cardiac injuries: clinical classification and management strategy. $\mathrm{Br} \mathrm{J}$ Surg. 1994;81:1572-5.

12. Karmy-Jones R, Namias N, Coimbra R, Moore EE, Schreiber $\mathrm{M}$, McIntyre $\mathrm{R}$, Croce $\mathrm{M}$, et al. Western trauma association critical decisions in trauma: penetrating chest trauma. J Trauma Acute Care Surg. 2014;77(6):994-1002.

13. Nicol AJ, Navsaria PH, Hommes M, Ball CG, Edu S, Kahn D. Sternotomy or drainage for a hemopericardium after penetrating trauma: a randomized controlled trial. Ann Surg. 2014;259(3):438-42.

14. Andrade AR, Mon L. Subxiphoid pericardial window in the diagnosis of penetrating cardiac trauma. Ann Thorac Surg. 1994;58:1139-41.

15. Navsaria PH, Nicol AJ. Haemopericardium in stable patients after penetrating injury: is subxiphoid pericardial window and drainage enough? A prospective study. Injury. 2005;36(6):745-50.

16. Gonza\#lez-Hadad A, OsornoMoncayo J, A\#ngel-Escobar I, Serna J, Salcedo A, Acero D, et al. Don't rush to a wide-open chest when diagnosing a hemopericardium in hemodynamically stable patients with penetrating injuries to the cardiac box. Presentado en el 77.. Congreso de la Asociacio\#n
Americana de Cirugi\#a de Trauma; 2018 sep; San Diego, Estados Unidos.

17. Chad TM, Namias N, Van Haren RM, Guarch GA, Ginzburg E, Salerno TA, et al. Does hemopericardium after chest trauma mandate sternotomy? J Trauma Acute Care Surg. 2012;72 (6):1518-24.

18. González-Hadad A, García AF, Serna JJ, Herrera MA, Morales M, Manzano-Núñez R. The role of ultrasound for detecting occult penetrating cardiac wounds in hemodynamically stable patients. World J Surg. 2020;44(5):1673-80.

19. Soares CB, Hoga LA, Peduzzi M, Sangaleti C, Yonekura T, Silva DR. Integrative review: concepts and methods used in nursing. Rev Esc Enferm USP. 2015;48(2):329-39. http://dx.doi.org/1 0.1590/S0080-6234201400002000020

20. Arom KV, Richardson JD, Webb G, Grover FL, Trinkle JK. Subxiphoid pericardial window in patients with suspected traumatic pericardial tamponade. Ann Thorac Surg. 1977;23 (6):545-9. https://doi.org /10.1016/s0003-4975(10)63699-5

21. Garrison N, Richardson D, Donald EF. Diagnostic transdiaphragmatic pericardiotomy in thoracoabdominal trauma. J Trauma. 1982;22(22);147-50.

22. Chestovich PJ, McNicoll CF, Fraser DR, Patel PP, Kuhls DA, Clark E, Fildes JJ. Selective use of pericardial window and drainage as sole treatment for hemopericardium from penetrating chest trauma. Trauma Surg Acute Care Open. 2018;3(1):e000187.

23. ATLS Subcommittee; American College of Surgeons' Committee on Trauma; International ATLS working group. Advanced trauma life support: 
the 10 edition. J Trauma Acute Care Surg. 2018:62-81.

24. Universidad de Antioquia, Departamento de Cirugía General. III Curso de Actualización en Cirugía General. 2019;3:28-39.

25. Tran H-V, Charles M, Garrett RC, Kempe PW, Howard CA, Khorgami Z. Ten-year trends in traumatic cardiac injury and outcomes: a trauma registry analysis. Ann Thorac Surg. 2020;110(3):844-8. https://doi.org/10. 1016/j.athoracsur.2019.12.038

26. Isaza-Restrepo A, Bolívar-Sáenz DJ, Tarazona-Lara M, Tovar JR. Penetrating cardiac trauma: analysis of 240 cases from a hospital in Bogota Colombia. World J Emerg Surg. 2017;12:26. https://doi.org/10.1186/s1 3017-017-0138-1

\section{Anexo}

\section{Anexo}

Escala de Clasificación de Heridas cardiacas de la Asociación Americana de Cirugía de Trauma

\begin{tabular}{|l|l|}
\hline Grado & \multicolumn{1}{|c|}{ Descripción } \\
\hline I & $\begin{array}{l}\text { Lesión cardiaca contusa con cambios menores en el ECG (cambios inespecíficos } \\
\text { de la onda T o del segmento ST, contracción atrial o ventricular prematura o } \\
\text { taquicardia sinusal persistente). } \\
\text { Lesión pericárdica contusa o penetrante sin lesión miocárdica, taponamiento o } \\
\text { herniación cardiaca. }\end{array}$ \\
\hline II & $\begin{array}{l}\text { Lesión cardiaca contusa con bloqueo eléctrico (bloqueo de rama izquierda o } \\
\text { derecha, fascicular anterior izquierda o atrioventricular) o con cambios } \\
\text { isquémicos (depresión del segmento ST o inversión de la onda T) sin falla } \\
\text { cardiaca. } \\
\text { Lesión miocárdica penetrante tangencial que no afecta el endocardio, sin } \\
\text { taponamiento cardiaco. }\end{array}$ \\
\hline III & $\begin{array}{l}\text { Lesión cardiaca contusa con más de } 6 \text { contracciones sostenidas o extrasístoles } \\
\text { ventriculares en un minuto. } \\
\text { Lesión cardiaca contusa o penetrante con ruptura septal, insuficiencia valvular } \\
\text { pulmonar o tricuspídea, disfunción de músculos papilares u oclusión distal de } \\
\text { alguna arteria coronaria sin falla cardiaca. } \\
\text { Laceración contusa pericárdica sin herniación cardiaca. }\end{array}$ \\
\hline IV & $\begin{array}{l}\text { Lesión contusa asociada a falla cardiaca. } \\
\text { taponamiecárdica tangencial penetrante que afecta el endocardio con } \\
\text { Lesión cardiaca contusa o penetrante con ruptura del tabique ventricular, } \\
\text { insuficiencia valvular pulmonar o tricuspídea, disfunción del músculo papilar u } \\
\text { oclusión de la arteria coronaria distal que produce falla cardiaca aguda. } \\
\text { Lesión cardiaca contusa o penetrante con insuficiencia valvular mitral o aórtica. } \\
\text { Lesión cardiaca contusa o penetrante del ventrículo derecho, aurícula derecha o } \\
\text { izquierda. }\end{array}$ \\
\hline VI & $\begin{array}{l}\text { Lesión cardiaca contusa o penetrante con oclusión proximal de alguna arteria } \\
\text { coronaria. } \\
\text { Lesión contusa o penetrante que con perforación del ventrículo izquierdo. } \\
\text { Herida estrellada con pérdida de menos del 50\% del tejido ventrícular derecho, } \\
\text { aurícula derecha o izquierda. }\end{array}$ \\
\hline $\begin{array}{l}\text { Lesión contusa que genera avulsión cardiaca. } \\
\text { Lesión penetrante que causa pérdida de más del } 50 \% \text { del tejido de alguna cámara } \\
\text { cardiaca. }\end{array}$ \\
\hline V
\end{tabular}

Fuente: Moore EE, Malangoni MA, Cogbill TH, Shackford SR, Champion HR, Jurkovich GJ, McAninch JW, Trafton PG. Organ injury scaling. IV: thoracic vascular, lung, cardiac, and diaphragm. J Trauma. 1994;36:299-300 